\title{
Bone-specific alkaline phosphatase flare in a patient with hepatitis c: case report and review of literature
}

\begin{abstract}
Introduction: Bone-specific alkaline phosphatase is a highly sensitive and specific marker of the bone-forming activity. Alkaline phosphatase (ALP) flare phenomenon is an acute elevation of serum ALP levels. ALP flare is thought to represent an exaggerated osteoblastic reaction.

Summary: A 53 year old man with Hepatitis $\mathrm{C}$ virus (HCV) infection had a sudden and significant elevation of BAP after treatment with sofosbuvir and ribavirin. Filgrastim treatments for leukopenia resulted in further elevation of ALP to 2323 IU/L. ALP levels steadily improved after discontinuation of $\mathrm{HCV}$ therapy and cessation of filgrastim injections.

Conclusion: ALP flare is a rare, severe elevation of ALP levels often induced by chemotherapeutic agents. We describe a unique case of ALP flare after treatment for HCV with sofosbuvir and ribavirin, further exacerbated by filgrastim therapy for leukopenia.

Keywords: alkaline phosphatase, sofosbuvir, ribavirin, hepatitis c, filgrastim, bone metabolism
\end{abstract}

Volume 4 Issue I - 2017

\author{
Silvia R Salgado Nunez del Prado, Elizabeth \\ M Lamos, Kashif M Munir \\ University of Maryland School of Medicine, USA
}

Correspondence: Kashif M Munir, Assistant Professor of Medicine, University of Maryland Center for Diabetes and Endocrinology, 827 Linden Avenue, 2nd floor, Baltimore, MD 2I20I, USA, Tel 443682 6800, Fax 410856 3840,

Emailkmunir@medicine.umaryland.edu

Received: October 28, 2016 | Published: January 18, 2017
Abbreviations: BAP, bone specific alkaline phosphatase; ALP, alkaline phosphatase; $\mathrm{HCV}$, hepatitis $\mathrm{C}$ virus; eGFR, estimated glomerular filtration rate; GGT, gamma glutamyl transferase; PTH, parathyroid hormone; Tc-99 MDP, technetium-99m methylene diphosphonate; CTX, C-telopeptide; CICP, C-terminal propeptide of type I collagen; OC, osteocalcin

\section{Introduction}

Bone-specific alkaline phosphatase (BAP) synthesized by osteoblasts is a highly sensitive and specific marker of the boneforming activity. Paget's disease is the most common cause of elevated serum BAP. Other causes include osteomalacia, rapid bone loss, bone growth, healing fracture, acromegaly, hyperthyroidism, osteogenic sarcoma, bone metastases, leukemia, myelofibrosis, and rarely myeloma. Alkaline phosphatase (ALP) flare phenomenon is an acute elevation of serum ALP levels. It has been described after chemotherapy initiation and is thought to represent an exaggerated osteoblastic reaction after treatment initiation. We describe the case of a patient with Hepatitis $\mathrm{C}$ virus (HCV) infection who had a sudden and significant elevation of BAP (ALP flare) and perform a review of the literature.

\section{Case presentation}

A 53-year-old male with orthotopic liver transplant in 2004 due to $\mathrm{HCV}$ cirrhosis was hospitalized for an acute on chronic elevationof serum ALP. Twenty-four weeks of sofosbuvir/ribavirin for the treatment of $\mathrm{HCV}$ was recently completed. He complained of fatigue, but denied other symptoms such as nausea, emesis, abdominal pain, jaundice, fever, bone or joint pain. Examination was notable for tenderness in the lower abdomen, but otherwise normal. Six weeks prior to admission, ALP was $235 \mathrm{IU} / \mathrm{L}$ and had risen to $1077 \mathrm{IU} / \mathrm{L}$ (nl: 39-117 IU/L). ALP isoenzyme showed a peak BAP of 95\% (nl: $12-68 \%$ ). Laboratory results Table 1 were notable for normal serum calcium, albumin, and liver functions. eGFR was reduced, GGT was mildly elevated 86U/L (nl: 15-73U/L), intact PTH was elevated 254pg/ $\mathrm{mL}$ (nl: $8-54 \mathrm{pg} / \mathrm{mL}$ ), and $25 \mathrm{OH}$ vitamin D was reduced $26.7 \mathrm{ng} / \mathrm{mL}$ (nl: $30-100 \mathrm{ng} / \mathrm{mL}$ ). Skeletal survey radiographs were normal. Tc-99 MDP bone scanshowed no scintigraphic evidence of osseous lesions or Paget's disease of bone. During his hospitalization, he received two filgrastim injections for leukopenia; a temporal relation between this medication and a further acute rise in ALP was observed on these two occasions, with a peak ALP of $2323 \mathrm{IU} / \mathrm{L}$ on hospital day 10. The level then decreased progressively to baseline after 5 months.

Table I Laboratory results

\begin{tabular}{lll}
\hline Prior to admission & \multicolumn{2}{c}{ ALP level (38- I 26 IU/L) } \\
\hline 90 days & \multicolumn{2}{c}{150} \\
45 days & \multicolumn{2}{c}{235} \\
7 days & \multicolumn{2}{c}{1077} \\
\hline Test & Result & Ref. Range \\
\hline WBC & 1.8 & $4.5-11.0 \mathrm{~K} / \mathrm{mcL}$ \\
Creatinine & 2.62 & $0.66-1.25 \mathrm{mg} / \mathrm{dL}$ \\
Calcium & 8.5 & $8.6-10.2 \mathrm{mg} / \mathrm{dL}$ \\
Albumin & 3.7 & $3.5-5.2 \mathrm{~g} / \mathrm{dL}$ \\
ALP & 965 & $38-126 \mathrm{units} / \mathrm{L}$ \\
AST & 33 & $10-59 \mathrm{units} / \mathrm{L}$ \\
ALT & 15 & $9-52 \mathrm{units} / \mathrm{L}$ \\
GGT & 86 & $15-73 \mathrm{U} / \mathrm{L}$ \\
BSAP & 271 & $0-20 \mathrm{ug} / \mathrm{L}$ \\
ALP-Isoenzymes & 1175 & $39-117 \mathrm{IU} / \mathrm{L}$ \\
Bone \% & 92 & $12-68 \%$ \\
Liver \% & 8 & $13-88 \%$ \\
\hline
\end{tabular}




\begin{tabular}{lll} 
Table continued... & & \\
\hline Test & Result & Ref. Range \\
\hline C-telopeptide & $240 \mathrm{I}$ & $87-345 \mathrm{pg} / \mathrm{mL}$ \\
PTH intact & 254 & $8-54 \mathrm{pg} / \mathrm{mL}$ \\
$25 \mathrm{OH}-$ Vitamin D & 26.7 & $30-100 \mathrm{ng} / \mathrm{mL}$ \\
I,25diOH-Vit D & 46.2 & $10-75 \mathrm{pg} / \mathrm{mL}$ \\
\hline
\end{tabular}

\section{Discussion}

This case exemplifies medication induced changes in ALP. This patient had a chronic elevation in ALP which likely represented a combination of liver associated elevation evidenced by the mild elevation in GGT and chronic vitamin D deficiency evidenced by the predominantly bone specific ALP percentage. However, the dramatic rise in ALP after ribavirin and filgastrim suggest that these medications were implicated in the observed ALP flare. The rise in BAP and c-telopeptide (CTX) reflects increased bone turnover.

BAP flare phenomena following HCV treatment has not been reported before. Low bone mass and osteoporosis are known complications of untreated chronic $\mathrm{HCV}$ infection with or without cirrhosis. ${ }^{1}$ These are a result of increased pro-inflammatory cytokines with subsequent exaggerated osteoclastogenesis. Before treatment, elevation of BAP and urine deoxypyridinoline excretion rate has been described, with subsequent normalization after treatment which may suggest a decrease in bone reabsorption. ${ }^{2}$

Ribavirin is a broad-spectrum antiviral agent used to treat a number of viral infections. In vitro, the effect of ribavirin is minimal on osteoblast proliferation or ALP activity. It significantly reduces osteoclast cell proliferation and increases cell death. No direct effect on osteoclast differentiation or function has been found, however it indirectly induces TRANCE/RANKL gene expression in osteoblasts, thus enhancing osteoclast formation. ${ }^{3-5}$ Solis-Herruzo et al., ${ }^{6}$ in 2000 reported that ribavirin use in combination with interferon alpharesulted in lowerbone mineral density (BMD) post-treatment which was significantly different when patients were treated with interferon alone. This suggests a potential role of ribavirin in bone loss, and was speculated to be secondary to impaired intestinal calcium absorption. However, several recent studies consistently describe an improvement on BMD after HCV treatment with interferon and ribavirin, which is likely due to a decrease in bone turnover as noted above. ${ }^{2,7,8}$ Hofmann et al., ${ }^{7}$ published the results of a prospective study on BMD and metabolism in patients with chronic HCV treated with pegylated interferon alpha and ribavirin. BMD, serum $\mathrm{C}$-terminal propeptide of type I collagen (CICP) and osteocalcin (OC) were obtained at baseline, after 48 weeks of therapy, and at the end of a 24-week followup period. Antiviral therapies resulted in a decrease in serum CICP and $\mathrm{OC}$ levels with subsequent increase of BMD. In contrast, a study that evaluated the effects of ribavirin on bone mineral metabolism in pediatric patients with chronic $\mathrm{HCV}$ treated with pegylated-interferon alpha- $2 \mathrm{~b}$ alone or combination of interferon and ribavirin showed no significant differences in $\mathrm{BMD}$, mean Z-scores between the groups before and after treatment. ${ }^{8}$ Serum, urinary biochemical values and bone markers were all normal in both groups, and no differences were observed pre and post-treatment.

Secondly, the patient received filgrastim, a recombinant granulocyte colony stimulating which stimulates the production and activity of neutrophils. In the initial studies on clinical toxicity, a twofold rise in ALP and a 2.5-fold increase with normal GGT levels was reported.
These changes were thought to result from myelogenous progenitor expansion within the bone marrow. ${ }^{9}$ Similarly, in a dose-escalation study of filgrastim versus placebo in patients with severe active rheumatoid arthritis, an increase in median ALP from baseline to day 5 was observed following administration..$^{10}$ Additionally, in a breast cancer study, 506 patients were randomized to chemotherapy with or without filgastrim at different doses. They found that serum levels of ALP were significantly higher in patients receiving filgrastim than those who did not $(\mathrm{p}=0.0001) .{ }^{11}$ Differentiation of ALP isoenzyme was not reported, however, there was no clinical or laboratory evidence of liver dysfunction, significant difference on transaminase levels or GGT elevation between both groups. Of note, ALP elevation resolved within 3 weeks.

The definitive roles of ribavirin and filgrastim on bone metabolism remain poorly understood. The historical increase in ALP observed after initiation of anti-viral treatment Figure 1 suggests that in this patient there was a negative effect of ribavirin on bone breakdown. Additionally, the dramatic increase in ALP after filgrastim administration, subsequent fall in levels, and identical pattern on second administration genuinely appears to reflect the direct action of filgrastim on ALP flare in this patient.

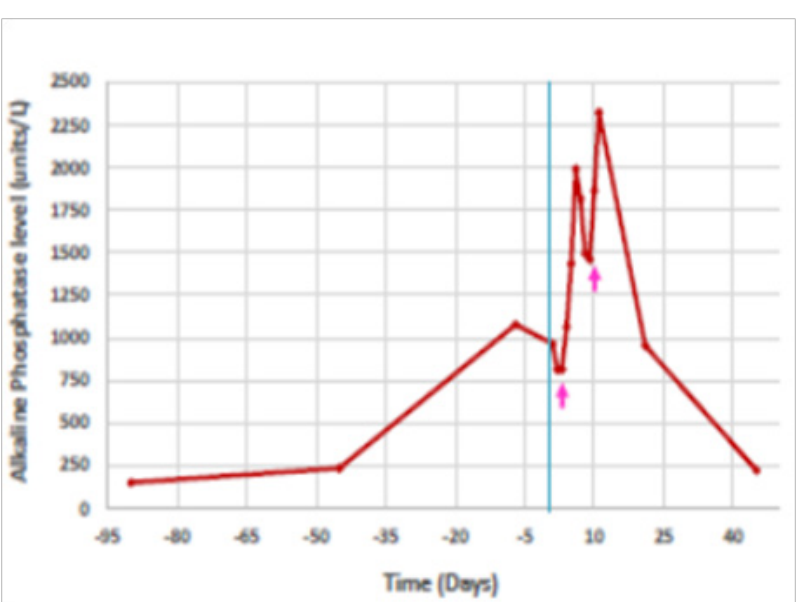

Figure I Alkaline phosphate trend before, during and after hospitalization. Arrow indicates G-CSF injections given for leucopenia.

\section{Conclusion}

In conclusion, this acute on chronic ALP flare demonstrated in the patient appears to be secondary to medication induced changes in bone metabolism. The underlying mechanism is not clear, but these effects appear to be self-limited. Although transient, this dramatic rise in ALP can result in significant imaging, laboratory evaluation and psychologic stress. A careful and thorough investigation of medication administration and timing identified the potential offending agents.

This patient's ALP eventually normalized to his pre-therapy baseline. He was additionally treated with cholecalciferol to treat his vitamin D deficiency.

\section{Acknowledgments}

None.

\section{Conflicts of interest}

The author declares there is no conflict of interest. 


\section{References}

1. Lai JC, Shoback DM, Zipperstein J, et al. Bone mineral density, bone turnover, and systemic inflammation in non-cirrhotics with chronic hepatitis C. Dig Dis Sci. 2015;60(6):1813-1819.

2. Redondo-Cerezo E, Casado-Caballero F, Martin-Rodriguez JL, et al. Bone mineral density and bone turnover in non-cirrhotic patients with chronic hepatitis and sustained virological response to antiviral therapy with peg-interferon alpha and ribavirin. Osteoporosis Int. 2014;25(6):1709-1715.

3. Bedimo R, Kang M, Tebas P, et al. Effects of Pegylated interferon/Ribavirin on bone turnover markers in HIV/Hepatitis C virus-coinfected patients. AIDS Res Hum retroviruses. 2016;32(4):325-328.

4. Moreira RO, Balduíno A, Martins HS, et al. Ribavirin, but not interferon alpha-2b, is associated with impaired osteoblast proliferation and differentiation in vitro. Calcif Tissue Int. 2004;75(2):160-168.

5. Lee J, Kim JH, Kim K, et al. Ribavirin enhances osteoclast formation through osteoblasts via up-regulation of TRANCE/RANKL. Mol Cell Biochem. 2007;296(1-2):17-24.

6. Solís-Herruzo JA, Castellano G, Fernández I, et al. Decreased bone mineral density after therapy with alpha interferon in combination with ribavirin for chronic hepatitis C. J Hepatol. 2000;33(5):812-817.
7. Hofmann WP, Kronenberger B, Bojunga J, et al. Prospective study of bone mineral density and metabolism in patients with chronic hepatitis $\mathrm{C}$ during pegylated interferon alpha and ribavirin therapy. J Viral Hepat. 2008;15(11):790-796.

8. Urganci N, Gulec SG, Arapoglu M, et al. The Effect of Ribavirin on Bone Density in Patients with Chronic Hepatitis C Treated with Interferon-Ribavirin Therapy. J Pediatr Gastroenterol Nutr. 2005;41(5):650652 .

9. Anderlini P, Przepiorka D, Seong D, et al. Clinical toxicity and laboratory effects of granulocyte-colony stimulating factor (filgrastim) mobilization and blood stem cell apheresis from normal donors, and analysis of charges for the procedures. Transfusion. 1996;36(7):590-595.

10. Snowden JA, Biggs JC, Milliken ST, et al. A randomized, blinded, placebo-controlled, dose escalation study of the tolerability and efficacy of filgrastim for haemopoietic stem cell mobilization in patients with severe active rheumatoid arthritis. Bone Marrow Transplant. 1998;22(11):1035-1041

11. Papaldo P, Di Cosimo S, Ferretti G, et al. Effect of Filgastrim on serum lactate dehydrogenase and alkaline phosphatase values in early breast cancer patients. Cancer Invest. 2004;22(4):650-653. 